\title{
Nonuniformly moving source in electromagnetic waveguides
}

\author{
VLADIMIR RABINOVICH, IVAN MIRANDA \\ Maestría en Ingeniería en Telecomunicaciones \\ Instituto Politécnico Nacional \\ ESIME Zacatenco \\ Edif. 1. Av. IPN, México D.F; 07738 \\ MÉXICO
}

Abstract: We consider the electromagnetic wave propagation generated by a nonuniformly moving source in homogeneous cylindrical waveguides. The asymptotic analysis of the problem with respect to a large parameter which simultaneously characterizes the slowness of changing of the amplitude and speed of the source is given.

\section{Introduction}

In the paper we consider the problem of electromagnetic wave propagation in the cylindrical waveguides with arbitrary cross-section generated by nonuniformly moving sources. This problem has a big theoretical and applied interest in the many branches of theoretical and applied physics, for example, in the satellite communications, nuclear and relativistic physics.

It should be noted that the problem of electromagnetic waves from moving electron is a classical problem of electrodynamics (see, for instance, [1]). The electromagnetic field from uniformly moving source in homogeneous waveguides was considered in the monograph [2], see, also papers devoted the wellknown Vavilov-Cherenkov effect in homogeneous waveguides generated by moving sources ( see, for instance, [4], [5], [3]).

We give here asymptotic solution of the problem with respect to a large parameter $\lambda \rightarrow+\infty$, which characterizes simultaneously the large distance between a moving source and a receiver, slowness of changing of the vertical speed of the source, and narrow-bandness of the amplitude of the source.

For asymptotic solution of the problem under consideration we use the representation of the field in the form of the double Fourier integral and use the stationary phase method. For acoustic problems of wave propagation in wave guides from moving source the indicated approach was used in the papers [7], [8], [9], [6].

\section{Statement of the problem}

We introduce the system of coordinates is in $\mathbf{R}^{3}$ as $\mathbf{r}=$ ( $\mathbf{x}, z)$ where $\mathbf{x}=\left(x_{1}, x_{2}\right)$. Let us consider a cylindrical waveguide,

$$
D=\left\{(\mathbf{x}, z) \in \mathbf{R}^{3}: \mathbf{x} \in \Omega, \mathrm{z} \in\{-\infty, \infty\}\right\}
$$

( $\varepsilon$ and $\mu$ constants) with an arbitrary cross-section and ideal boundary.

We consider a source of electromagnetic vibrations moving inside the waveguide with the following trajectory,

$$
\mathbf{r}=\mathbf{r}_{0}(t)=\left(x_{1}^{0}(t), x_{2}^{0}(t), z_{0}(t)\right), t \in \mathbf{R} .
$$

In what follows we suppose that $\mathbf{v}(t)=\dot{r}^{0}(t) \neq 0$ for each $t$.

The sources of electromagnetic vibrations are described as

$$
\begin{aligned}
& \rho(\mathbf{r}, t)=A(t) \delta\left(\mathbf{r}-\mathbf{r}_{0}(t)\right) \\
& \mathbf{J}(\mathbf{r}, t)=A(t) \mathbf{v}(t) \delta\left(\mathbf{r}-\mathbf{r}_{0}(t)\right)
\end{aligned}
$$

where $\rho(\mathbf{r}, t)$ and $\mathbf{J}(\mathbf{r}, t)$ are the charge and current densities, and both are related by means of the continuity equation

$$
\nabla \cdot \mathbf{J}+\frac{\partial \rho}{\partial t}=0
$$

In what follows we consider the propagation of transverse electric (TE) and transverse magnetic (TM). 
More specifically, for TE-waves the electric field is transverse to the direction of the propagation. For TMwaves, the magnetic field is transverse to the direction of the propagation. By means of the TE and TM waves, we can describe the $B_{z}$ and $E_{z}$ components, respectively.

It is well known that $H_{\mathrm{z}}$ and $E_{\mathrm{z}}$ satisfy the following equations,

$$
\begin{aligned}
& \frac{1}{c^{2}} \frac{\partial^{2}}{\partial t^{2}} H_{z}-\nabla_{\mathbf{x}}^{2} H_{z}-\frac{\partial^{2}}{\partial z^{2}} H_{z}=-\left(\frac{\partial J_{2}}{\partial x_{1}}-\frac{\partial J_{1}}{\partial x_{2}}\right) \\
& \frac{1}{c^{2}} \frac{\partial^{2}}{\partial t^{2}} E_{z}-\nabla_{\mathbf{x}}^{2} E_{z}-\frac{\partial^{2}}{\partial z^{2}} E_{z}=-\frac{1}{c^{2}}\left(J_{z}\right)^{\prime}-\frac{\partial \rho}{\partial z}
\end{aligned}
$$

where $c=\frac{1}{\sqrt{\mu \varepsilon}}$ is the speed of the light in the media.

For the waveguides with the ideal boundary $\partial D$ we have the following boundary conditions: $\left.E_{z}\right|_{\partial D}=0,\left.\frac{\partial H_{z}}{\partial z}\right|_{\partial D}=0$.

\section{Deriving of the asymptotic of the fields generated by moving sources}

\subsection{Green Function for waveguides}

The Green function $g_{E}=g_{E}\left(\omega, \mathbf{x}, \mathbf{x}^{0}, z\right)$ for the electric waves in the wave guide $\mathrm{D}$ is the solution of the boundary value problem for the Helmholtz equation:

$$
\begin{aligned}
& \nabla_{\mathbf{x}}^{2} g_{E}-\frac{\omega^{2}}{c^{2}} g_{E}-\frac{\partial^{2} g_{E}}{\partial z^{2}}=\delta\left(\mathbf{x}-\mathbf{x}^{0}\right) \delta(z), \\
& (\mathbf{x}, z) \in D,\left.g_{E}\right|_{\partial D}=0
\end{aligned}
$$

The following spectral problems are connected with these equations:

$$
\nabla_{\mathbf{x}}^{2} \varphi=\lambda_{j} \varphi_{j}, x \in M,\left.\varphi\right|_{\partial M}=0,
$$

It is well known that the spectral problem (6), have a discreet spectrum $\left\{\lambda_{j}\right\}_{j=1}^{\infty}, \lambda_{j} \leq 0$, and the orthonormal in $L_{2}(M)$ system $\left\{\varphi_{j}\right\}_{j=1}^{\infty}$ of eigenfunctions which are complete in the space $L_{2}(M)$. Let $\lambda_{j}=-\mu_{j}^{2}$. Then it is well-known that the Green function has the following representations

$$
g_{E}\left(\omega, \mathbf{x}, \mathbf{x}^{0}, z\right)=\sum_{j=1}^{\infty} \frac{\varphi_{j}\left(\mathbf{x}^{0}\right) \varphi_{j}(\mathbf{x}) e^{i \gamma_{j}(\omega)|z|}}{2 i \gamma_{j}(\omega)},
$$

$$
\text { where } \gamma_{j}(\omega)=\sqrt{\frac{\omega^{2}}{c^{2}}-\mu_{j}^{2}} \text {. }
$$

The terms in (7) are called the modes, and if $j$ is such that $\gamma_{j}(\omega) \geq 0$ the mode is called propagated, in the opposite case they are called no propagated. The no propagated modes are not interesting in the problem of wave propagation on long distances.

\subsection{Asymptotic of the $E_{z}$-component}

The components $E_{z}$ for the case of the moving source satisfies the following equation

$$
\frac{1}{c^{2}} \frac{\partial^{2}}{\partial t^{2}} E_{z}-\nabla_{\mathbf{x}}^{2} E_{z}-\frac{\partial^{2}}{\partial z^{2}} E_{z}=-\frac{1}{c^{2}}\left(J_{z}\right)_{t}^{\prime}-\frac{\partial \rho}{\partial z}
$$

where

$$
\begin{aligned}
& J_{z}(\mathbf{r}, t)=A(t) z^{0}(t) \delta\left(\mathbf{r}-\mathbf{r}^{0}(t)\right) \\
& \rho(\mathbf{r}, t)=A(t) \delta\left(\mathbf{r}-\mathbf{r}^{0}(t)\right)
\end{aligned} .
$$

Let us consider the case when $A(t)$ and $\mathbf{r}^{0}(t)=\left(x_{1}^{0}(t), x_{2}^{0}(t), z^{0}(t)\right)$ depend on the large parameter $\lambda>1$. We suppose that

$$
\begin{aligned}
& A(t)=a(t / \lambda), x_{1}^{0}(t)=X_{1}^{0}(t, \lambda) \\
& x_{2}^{0}(t)=X_{2}^{0}(t / \lambda), z^{0}(t)=\lambda Z^{0}(t, \lambda)
\end{aligned}
$$

where $a(t), X_{1}^{0}(t), X_{2}^{0}(t), Z^{0}(t)$ are two times differentiable on $\mathbf{R}$ functions bounded with its derivatives. The dependence in (8), (9) on the large parameter $\lambda$ means that the amplitude $A(t)$ is slowly varying, the vertical component of the speed is small, and the horizontal acceleration is small if the parameter $\lambda$ is large enough.

In what follows we suppose that $\sup _{t \in \mathbf{R}}|v(t)|<c$, that is the source moves with a sub-light velocity. 
Applying the Fourier transform with respect $t$ we obtain a representation of $E_{z}(t, \mathbf{x}, z)$ in the form of the double Fourier integral with respect the time and the frequency and neglecting by the members with order $O(1 / \lambda), \lambda \rightarrow \infty$ we obtain that

$E_{z}(t, \mathbf{x}, z)=\frac{1}{2 \pi} \int_{-\infty}^{\infty} \int_{-\infty}^{\infty} e^{-i \omega(t-\tau)} B(\tau / \lambda) \times$

$\left(g_{E}\right)_{z}^{\prime}\left(\omega, \mathbf{x}, \mathbf{X}^{0}(\tau, \lambda), z-\lambda Z_{0}(\tau / \lambda) d \omega d \tau(1+O(1 / \lambda))\right.$

where $B(\tau)=a(\tau)\left(\frac{\left(Z_{0}^{\prime}\right)^{2}(\tau)}{c^{2}}-1\right)$. Applying the decomposition for the Green function $g_{E}$ we obtain that

$$
\begin{aligned}
& E_{z}(t, \mathbf{x}, z)=\frac{1}{4 \pi} \sum_{j=1}^{\infty} \int_{-\infty-\infty}^{\infty} \int_{-\infty}^{\infty} e^{-i \omega(t-\tau)} B(\tau / \lambda) e^{-i \omega_{0} \tau} \times \\
& \varphi_{j}\left(\mathbf{X}^{0}(\tau / \lambda)\right) \varphi_{j}(\mathbf{x}) e^{i \gamma_{j}(\omega)\left|z-\lambda Z_{0}(\tau / \lambda)\right|} \times \\
& \operatorname{sgn}\left(z-\lambda Z_{0}(\tau / \lambda)\right) d \omega d \tau
\end{aligned}
$$

Let us introduce new variables $z=\lambda Z, t=\lambda T$. Making the change of variable in (10), $\tau^{\prime}=\tau / \lambda$ we obtain the representation

$$
E_{z}(T / \lambda, \mathbf{x}, Z / \lambda)=\sum_{j=1}^{\infty} E_{z}^{(j)}(T / \lambda, \mathbf{x}, Z / \lambda)
$$

where

$$
\begin{aligned}
& E_{z}^{(j)}(T / \lambda, \mathbf{x}, Z / \lambda)=\frac{\lambda}{4 \pi} \int_{-\infty-\infty}^{\infty} \int_{-\infty}^{\infty} e^{i \lambda \omega\left(T-\tau^{\prime}\right)} B\left(\tau^{\prime}\right) \times \\
& e^{-i \lambda \omega_{0} \tau^{\prime}} \varphi_{j}\left(\mathbf{X}^{0}\left(\tau^{\prime}\right)\right) \varphi_{j}(\mathbf{x}) e^{i \lambda \gamma_{j}(\omega)\left|Z-Z 0\left(\tau^{\prime}\right)\right|} \times \\
& \operatorname{sgn}\left(Z-Z_{0}\left(\tau^{\prime}\right)\right) d \omega d \tau^{\prime}(1+O(1 / \lambda))
\end{aligned}
$$

The next step is the asymptotic analysis of $E_{z}^{(j)}(T / \lambda, \mathbf{x}, Z / \lambda)$ if $\lambda \rightarrow+\infty$. For asymptotic analysis of modes $E_{z}^{(j)}(T / \lambda, \mathbf{x}, Z / \lambda)$ we apply the stationary phase method (see, for instance [10]).

The phase in the integral (12) is

$$
\widetilde{S}_{j}\left(\omega, \tau^{\prime}\right)=\gamma_{j}(\omega)\left|Z-Z_{0}\left(\tau^{\prime}\right)\right|-\omega\left(T-\tau^{\prime}\right)-\omega_{0} \tau^{\prime}
$$

The stationary point $\left(\omega_{0 j}, \tau_{0 j}^{\prime}\right)$ of the phase $\widetilde{S}_{j}\left(\omega, \tau^{\prime}\right)$ is the solution of the following system

$$
\begin{aligned}
\widetilde{S}_{j \omega}^{\prime}\left(\omega, \tau^{\prime}\right)= & \gamma_{j}^{\prime}(\omega)\left|Z-Z_{0}\left(\tau^{\prime}\right)\right|-\left(T-\tau^{\prime}\right)=0 \\
\widetilde{S}_{j \tau^{\prime}}^{\prime}\left(\omega, \tau^{\prime}\right)= & -\gamma_{j}(\omega) Z_{0}^{\prime}\left(\tau^{\prime}\right) \operatorname{sgn}\left(Z-Z_{0}\left(\tau^{\prime}\right)\right) . \\
& +\omega-\omega_{0}=0
\end{aligned}
$$

The Hess matrix of the phase $\widetilde{S}_{j}\left(\omega, \tau^{\prime}\right)$ is of the form

$$
\widetilde{S}^{\prime \prime}\left(\omega, \tau^{\prime}\right)=\left(\begin{array}{ll}
\frac{\partial^{2} \widetilde{S}^{\prime \prime}}{\partial \omega^{2}} & \frac{\partial^{2} \widetilde{S}^{\prime \prime}}{\partial \omega \partial \tau^{\prime}} \\
\frac{\partial^{2} \widetilde{S}^{\prime \prime}}{\partial \omega \partial \tau^{\prime}} & \frac{\partial^{2} \widetilde{S}^{\prime \prime}}{\partial \tau^{\prime 2}}
\end{array}\right) .
$$

One can show that in the case of a sub-light move of the source the Hess matrix is not degenerated $\left(\operatorname{det} \widetilde{S}^{\prime \prime}\left(\left(\omega_{0 j}, \tau_{0 j}^{\prime}\right)\right) \neq 0\right)$. Then it follows from the stationary phase method that $E_{z}^{(j)}(T / \lambda, \mathbf{x}, Z / \lambda)$ has the following asymptotic

$$
\begin{gathered}
E_{z}^{(j)}(T / \lambda, \mathbf{x}, Z / \lambda)=\frac{1}{2} B\left(\tau_{0 j}^{\prime}\right) \varphi_{j}\left(\mathbf{X}_{0}\left(\tau_{0 j}^{\prime}\right)\right) \times \\
\varphi_{\mathrm{j}}(\mathbf{x}) \operatorname{sgn}\left(\mathrm{z}-\lambda Z_{0}\left(\tau_{0 \mathrm{j}}^{\prime}\right)\right) \times \\
e^{i \lambda\left(\gamma_{j}\left(\omega_{0 j}\right)\left|Z-Z_{0}\left(\tau_{0 j}^{\prime}\right)\right|-\omega_{0}\left(T-\tau_{0 j}^{\prime}\right)-\omega_{0} \tau_{0 j}^{\prime}\right)} \times \\
e^{+\operatorname{det} \widetilde{S}^{\prime \prime}\left(\omega_{0 j}, \tau_{0 j}^{\prime}\right)} \times
\end{gathered}
$$

where $\operatorname{sgn} \widetilde{S}^{\prime \prime}\left(\omega_{0 j}, \tau_{0_{j}}^{\prime}\right)$ is the difference between the number of the positive and negative eigenvalues of the Hess matrix.

Returning to the coordinates $t=\lambda T, \quad z=\lambda Z$, $\tau_{0 j}=\lambda \tau_{0 j}^{\prime}$ we obtain that

$$
E_{z}(t, \mathbf{x}, z) \approx \sum_{j=1}^{N} E_{z}^{(j)}(t, \mathbf{x}, z),
$$

where 


$$
\begin{aligned}
& E_{z}^{(j)}(t, \mathbf{x}, z)=A\left(\tau_{0 j}\right)\left(\frac{z_{0}^{\prime 2}\left(\tau_{0 j}\right)}{c^{2}}-1\right) \varphi_{j}\left(\mathbf{x}^{0}\left(\tau_{0 j}\right)\right) \times \\
& \varphi_{j}(\mathbf{x}) \frac{e^{i \lambda\left(\gamma_{j}\left(\omega_{0 j}\right)\left|z-z_{0}\left(\tau_{0 j}\right)\right|-\omega_{0 j}\left(t-\tau_{0 j}\right)-\omega_{0 j} \tau_{0 j}\right)}}{2 \sqrt{\operatorname{det} S^{\prime \prime}\left(\omega_{0 j}, \tau_{0 j}\right)}} \times \\
& e^{+i \frac{\pi}{4} \operatorname{sgn} S^{\prime \prime}\left(\omega_{0 j}, \tau_{0 j}\right)}(1+O(1 / \lambda))
\end{aligned}
$$

and $\left(\omega_{0 j}, \tau_{0 j}\right)$ is a stationary point of the phase

$$
S_{j}(\omega, \tau)=\gamma_{j}(\omega)\left|z-z_{0}(\tau)\right|-\omega(t-\tau)-\omega_{0} \tau,
$$

which is defined as a solution of the system

$$
\begin{aligned}
S_{j \omega}^{\prime}(\omega, \tau)= & \gamma_{j}^{\prime}(\omega)\left|z-z_{0}(\tau)\right|-(t-\tau)=0 \\
S_{j \tau}^{\prime}(\omega, \tau)= & -\gamma_{j}(\omega) z_{0}^{\prime}(\tau) \operatorname{sgn}\left(z-z_{0}(\tau)\right) . \\
& +\omega-\omega_{0}=0
\end{aligned}
$$

It should be noted that only a finite number $\mathrm{N}$ of members in the sum (11) have the stationary points of the phase, thus summation in formula (13) is taken with respect to a finite number of the mode such that the stationary phase $S_{j}(\omega, \tau)$ has stationary points.

The parameter $\lambda$ has the following physical meaning. If $\lambda \rightarrow \infty$ the distance between the moving source and the receptor tends to infinity.

This parameter also characterizes the slowness of changing of the amplitude and smallness of vertical components of the speed with respect to horizontal one.

\subsection{Asymptotic of the transversal components}

Let

$\mathbf{E}_{j}^{\prime(\lambda)}\left(t, \mathbf{x}^{\prime}, z\right)=\frac{i \omega_{0 j}(t) \varepsilon}{\gamma_{j}^{2}\left(\omega_{0 j}(t)\right)} \nabla^{\prime} \times H_{z j}^{(\lambda)}\left(t, \mathbf{x}^{\prime}, z\right)$,

where $\mathbf{H}_{j}^{\prime(\lambda)}=\left(0,0, H_{z j}^{(\lambda)}\right), \nabla^{\prime}=\left(\frac{\partial}{\partial x_{1}}, \frac{\partial}{\partial x_{2}}, 0\right)$

and

$$
\mathbf{H}_{j}^{\prime(\lambda)}\left(t, \mathbf{x}^{\prime}, z\right)=\frac{\varepsilon \mu^{-1}}{\gamma_{j \mu}^{2}\left(\omega_{0 j}(t)\right)} \frac{\partial}{\partial z}\left(\nabla^{\prime} H_{j z}^{(\lambda)}\left(t, \mathbf{x}^{\prime}, z\right)\right) .
$$

Then the transversal components of the electromagnetic waves radiated by moving source are given by the formulas

$$
\begin{aligned}
\mathbf{E}^{\prime(\lambda)}\left(t, \mathbf{x}^{\prime}, z\right) & =\sum_{j=1}^{N} \mathbf{E}_{j}^{\prime(\lambda)}\left(t, \mathbf{x}^{\prime}, z\right), \\
\mathbf{H}^{\prime(\lambda)}\left(t, \mathbf{x}^{\prime}, z\right) & =\sum_{j=1}^{N} \mathbf{H}_{j}^{\prime(\lambda)}\left(t, \mathbf{x}^{\prime}, z\right),
\end{aligned}
$$

where $\mathbf{E}_{j}^{\prime(\lambda)}\left(t, \mathbf{x}^{\prime}, z\right), \quad \mathbf{H}_{j}^{\prime(\lambda)}\left(t, \mathbf{x}^{\prime}, z\right)$, are defined by (14) and (15).

\subsection{Doppler shift and retarded time}

We can obtain explicit expressions for the Doppler shift and retarded time for the mode components of the electromagnetic field.

Let

$$
S_{j}(\omega, \tau)==\gamma_{j}(\omega)\left|\mathbf{x}^{\prime}-\mathbf{x}^{0^{\prime}}(\tau)\right|-\omega(t-\tau)-\omega_{0} \tau
$$

be the phase of the mode with number $j$. This phase depends on the time $t$ in the explicit form. It is convenient this dependence to write as

$$
S_{j}(\omega, \tau)=\hat{S}_{j}(\omega, \tau, t) .
$$

Let $\left(\omega_{0 j}(t), \tau_{0 j}(t)\right)$ be a stationary point of this phase. Then the instantaneous frequency is given by the formula

$$
\omega_{j}^{(i n)}(t)=-\frac{d \hat{S}_{j}\left(\omega_{0 j}(t), \tau_{j}(t), t\right)}{d t} .
$$

Because $\left(\omega_{0 j}(t), \tau_{0 j}(t)\right)$ be a stationary point of the phase $\hat{S}_{j}(\omega, \tau, t)$ we obtain that $\omega_{j}^{(i n)}(t)=\omega_{0 j}(t)$. Hence the instantaneous frequency coincides with the first component of the stationary point.

Thus we obtain the explicit formula for the mode Doppler effect

$$
\Delta_{j}(t)=\omega_{0 j}(t)-\omega_{0} .
$$


It is easy to see that $\tau_{j}(t)$ is the retarded time for the mode with number $j$, that is the time of the radiation of the signal for which the mode with number $j$ arrives to the receiver at the moment $t$.

Thus the stationary phase point $\left(\omega_{0 j}, \tau_{0 j}\right)$ has the following physical meaning: is an instantaneous frequency and $\tau_{0 j}$ is a retarded time for the mode with number $j$, and $\Delta \omega_{j}=\omega_{0}-\omega_{0 j}$ is the Doppler effect for the frequency, and $\Delta t_{j}=t-\tau_{0 j}$ is the Doppler effect for the time.

\section{Moving source in a rectangular wave guide}

Let us consider a rectangular wave guide, the trajectory of the source is

$$
\begin{aligned}
& \mathbf{r}=\mathbf{r}_{0}(t)=\left(x_{1}^{0}(t), x_{2}^{0}(t), v t\right), 0<v<c \\
& 0<x_{1}^{0}(t)<a, 0<x_{2}^{0}(t)<b
\end{aligned}
$$

where $x(t)_{1}^{0}, x_{2}^{0}(t)$ depend on the parameter $\lambda$ as above.

The coordinates of the receptor are $\mathbf{r}=\left(x_{1}, x_{2}, 0\right)$, where $0<x_{1}<a, 0<x_{2}<b$. In this case $z_{0}(t)=v t, z_{0}^{\prime}(t)=v$.

The phase is

$$
S_{m n}(\omega, \tau)=\gamma_{m n}(\omega)|v \tau|-\omega(T-\tau)-\omega_{0} \tau
$$

where

$$
\gamma_{m n}(\omega)=\sqrt{k^{2}-\left(\frac{m \pi}{a}\right)^{2}-\left(\frac{n \pi}{b}\right)^{2}, k^{2}=\frac{\omega^{2}}{c^{2}} .}
$$

The group velocity of the mode with number $m n$ is given by

$$
U_{m n}(\omega)=\frac{1}{\gamma_{m n}^{\prime}(\omega)}=\frac{c^{2}}{\omega} \sqrt{\frac{\omega^{2}}{c^{2}}-\left(\frac{m \pi}{a}\right)^{2}-\left(\frac{n \pi}{b}\right)^{2}} .
$$

The point of stationary phase is a solution of the following system:

$$
\begin{aligned}
& \left(S_{m n}\right)_{\omega}^{\prime}(\omega, \tau)=\frac{|v \tau|}{U_{m n}(\omega)}-t+\tau=0 \\
& \left(S_{m n}\right)_{\tau}^{\prime}(\omega, \tau)=\gamma_{m n}(\omega)|v| \operatorname{sgn} \tau+\omega-\omega_{0}=0
\end{aligned}
$$

Let $v>0$ and $\left(\omega_{m n}, \tau_{m n}\right)$ be a solution of the system under consideration. Then

$$
\tau=\frac{t}{1+\frac{v}{U_{m n}(\omega)}}>t>0
$$

and $\omega_{m n}$ is a solution of the quadratic equation

$$
\left(\omega-\omega_{0}\right)^{2}=\left(\gamma_{m n}(\omega) v\right)^{2} .
$$

The solutions of these equations are

$$
\omega_{m n}=\frac{\omega_{0} \pm \sqrt{\frac{\omega_{0}^{2}}{c^{2}}-\left(1-\frac{v^{2}}{c^{2}}\right)\left(\left(\frac{m \pi}{a}\right)^{2}+\left(\frac{n \pi}{b}\right)^{2}\right)}}{\left(1-\frac{v^{2}}{c^{2}}\right)}
$$

The formula for $\left(S_{m n}\right)_{\tau}^{\prime}(\omega, \tau)$ implies that $\omega>\omega_{0}$. Then we should take plus in the last formula.

Then for this case, the point of stationary phase $\left(\omega_{m n}, \tau_{m n}\right)$ is found in an explicit form.

The Hess matrix for this case has a triangle form.

$$
S_{m n}^{\prime \prime}\left(\omega_{m n}, \tau_{m n}\right)=\left(\begin{array}{cc}
0 & \left(1-\frac{v^{2}}{U_{m n}\left(\omega_{m n}\right)}\right) \\
\left(1-\frac{v^{2}}{U_{m n}\left(\omega_{m n}\right)}\right) & \gamma_{m n}^{\prime \prime}(\omega)\left|v \tau_{m n}\right|
\end{array}\right) .
$$

Moreover, $\operatorname{det} S_{m n}^{\prime \prime}\left(\omega_{m n}, \tau_{m n}\right)=-\left(1-\frac{v^{2}}{U_{m n}\left(\omega_{m n}\right)}\right)^{2}$ and

$$
\operatorname{sgn} S_{m n}^{\prime \prime}\left(\omega_{m n}, \tau_{m n}\right)=0
$$

We have obtained the asymptotic formula for the field in the rectangular waveguide: 


$$
\begin{aligned}
& E_{z}^{(\lambda)}(t, \mathbf{x}, z)=\sum_{1}^{N\left(\omega_{0}\right)} \frac{2}{a b} A\left(\tau_{m n}\right) \sin \frac{m \pi x_{1}}{a} \sin \frac{n \pi x_{2}}{b} \times \\
& \sin \frac{m \pi x_{10}\left(\tau_{m n}\right)}{a} \sin \frac{n \pi x_{20}\left(\tau_{m n}\right)}{b} \times \\
& \frac{\exp \left(i \gamma_{m n}\left(\omega_{m n}\right) v \tau_{m n}-\omega\left(t-\tau_{m n}\right)-\omega_{0} \tau_{m n}\right)}{2 i \gamma_{m n}\left(\omega_{m n}\right) \mid 1-\frac{v}{U_{m n}\left(\omega_{m n}\right) \mid}} .
\end{aligned}
$$

The mode Doppler effect produced by the moving sources is given by

$$
\Delta_{m n} \omega_{0}=\omega_{m n}-\omega_{0}, \Delta_{m n} t=\tau_{m n}-t
$$

The formula for $E_{z}^{(\lambda)}(t, \mathbf{x}, z)$ is very simple and convenient for the numerical analysis.

\subsection{Numerical Calculations}

Let us consider as an example a rectangular wave guide with the following characteristics: $a=b=10 \mathrm{~m}$, $\varepsilon=(1 / 36 \pi) \times 10^{-9} \mathrm{~F} / \mathrm{m}$ and $\mu=4 \pi \times 10^{-7} \mathrm{H} / \mathrm{m}$, $\mathbf{r}^{0}(t)=(5,5, v t), v=3070 \mathrm{~m} / \mathrm{s}, \omega_{0}=3 \times 10^{8} \mathrm{rad} / \mathrm{s}$. The receiver is located at the point $r=(5,5,0)$. The behavior of $\left|E_{z}\right|$ as the function of the time is given by the following graphic:

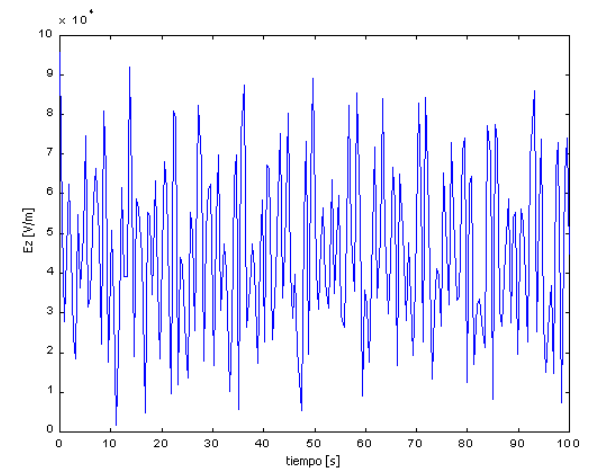

Fig. 1. Behavior of the field for the speed of 3070 $\mathrm{m} / \mathrm{s}$

Next graphic shows the effect of the speed in the behavior of the field, in this case the speed of the source is $200 \mathrm{~m} / \mathrm{s}$.

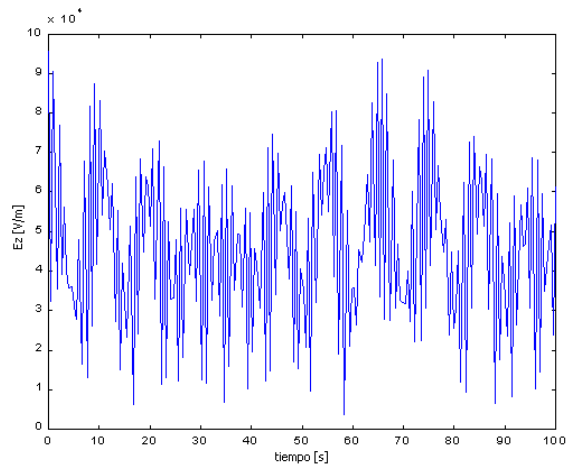

Fig. 2. Behavior of the field for the speed of $200 \mathrm{~m} / \mathrm{s}$

The graphics demonstrate a dependence of the field structure from the speed of the source.

References:

[1] L.D. Landau, E.M. Lifschitz, Field Theory, M. Nauka, 1988.

[2] L.B. Felsen, N. Marcuvitz, Radiation and Scattering of Waves, Prentice Hall, New Jersey, 1973.

[3] Bolotovskiy B.M; Ginzburg V.D. The effect of Vavilov-Cherenkov and Doppler effect under moving sources with velocity larger than light speed in the vacuum. Uspehi fiz. nauk, 1972, v.6, No. 4.

[4] S.V. Afanas'ev, B.M. Bolotovskiy, On radiation of supertight sources in waveguides. Short Communications in Physics, M:FIAN, 1972, No.10, p.29.

[5] E.A. Begloyan, S.X. Bekova, On supertight source in waveguides, Izv. AN SSSR, Fizika, 1979, v.14, No.4, p.240-260

[6] V.I.Il'ichev, V.S. Rabinovich, E.A. Rivelis, U.V. Hoha, Acoustic field of a moving narrow-band source in oceanic waveguides, Dokl. Akad. Nauk SSSR, 304 (1989), No. 5, pp.1123-1127 (in Russian).

[7] O.A. Obrezanova, V.S. Rabinovich, Acoustic field of a source moving along stratified waveguide surface, Akusticheskii Jurnal, 39 (1993), No. 3, pp.517-521 (in Russian).

[8] S.M. Grudsky, O.A. Obrezanova, V.S. Rabinovich, Sound propagation from a moving air source in the ocean covered by ice, Akusticheskii Jurnal, 41 (1995), No.6, pp. 1-6 (in Russian)

[9] O.A. Obrezanova, V.S. Rabinovich, Acoustic field, generated by moving source in stratified waveguides. Wave Motion, 27 (1998), 155-167.

[10] M.V. Fedoryk, The saddle-point method, Moscow, Nauka, (1977) (in Russian). 